\title{
La Búsqueda de Interacción Semántica en la Web de las Cosas: Una Revisión
}

INVESTIGACIÓN E INNOVACIÓN en INGENERÍAS ISSN2344-8652

OPEN A ACCESS

Recibido: 22/09/2020

Aceptado: 22/10/2020

Publicado: 23/11/2020

Correspondencia de autores: manzamb@unicauca.edu.co

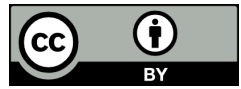

Copyrith 2020

by Investigación e Innovación en Ingenierías

\section{The Search for Semantic Interaction in the Web of Things: Review}

Miguel Niño Zambrano

Jovani Alberto Jiménez Builes

Universidad Nacional de Colombia
Gustavo Ramírez González

Universidad del Cauca, Colombia de

de

ello

.

\begin{abstract}
Resumen
Objetivo: Presentar un estado del arte alrededor de problemas que surgen al crear servicios complejos de interacción entre sensores en la Web, como: descubrimiento automático, combinación de datos heterogéneos, coordinación y cooperación entre sensores, solucionados con el desarrollo e middlewares semánticos para la Web de las Cosas, sin lograr una cooperación transparente entre llos, profundizando en aportes en la interacción máquina a máquina. Metodología: La investigación fue de tipo documental a través de un análisis bibliográfico de los últimos 12 años, identificando núcleos temáticos, criterios de selección, fichas descriptivas y realizando abstracción de estas para su desarrollo. Resultados y conclusiones: Los resultados exhiben por núcleo temático las principales componentes del tema, permitiendo una discusión alrededor de los retos por resolver en esta área de investigación, analizando enfoques presentes en el estado actual del conocimiento relacionados con la interacción semántica de la Web de las cosas (WoT).
\end{abstract}

Palabras clave: Web semántica de objetos, objetos inteligentes, interacción semántica, contexto, ontologías para la wot.

Abstract

Objective: T: Present a state of the art around problems that arise when creating complex interaction services between sensors on the web, such as: automatic discovery, combination of heterogeneous data, coordination and cooperation between sensors, solutions with the development of semantic middlewares for the web of Things, without achieving transparent cooperation between them, deepening contributions in machine-to-machine interaction. Methodology: The research was of a documentary type through a bibliographic analysis of the last 12 years, identifying thematic nuclei, selection criteria, descriptive sheets, abstracting them for their development. Results and conclusions: The results exhibited by thematic core the main components of the topic, problems a discussion around the challenges by resolution in this research area, analyzing current approaches in the current state of knowledge related to the semantic interaction of the Web of things (WoT).

Keywords: Semantic web of objects, smart objects, semantic interaction, context, ontologies for wot.

Como citar (IEEE): M. Niño-Zambrano., G. Ram'rez-González., A. Muñoz-Velasco., J. Jiménez-Builes., y E. Granados-pemberty. “La Búsqueda de Interacción Semántica en la Web de las Cosas: Una Revisión”, vol. 8, n³, pp. 72-84, 2020. DOI: https://doi.org/10.17081/ invinno.8.3.4705 


\section{Introducción}

Las propuestas realizadas para la interacción semántica de objetos en la Web de las cosas (Web of things, WoT), que corresponde a la capacidad de dos o más objetos inteligentes de la WoT para comunicarse entre sí en un contexto bien definido [1], exploran lo que se ha llamado el "Social Web of Things" [2,3], como una estrategia para compartir información entre objetos y humanos acerca de los datos de los sensores, el contexto de operación y otros servicios de alto nivel. Los primeros trabajos se centraron en como conectar los objetos a las redes sociales existentes [4,5], hacia la interacción de las "cosas" con los usuarios y no a la interacción entre las "cosas" mismas; otros, se enfocaron en conectar salidas con entradas de dispositivos [6], necesitando aún la intervención de expertos para definir las conexiones. Los trabajos más recientes proponen arquitecturas sociales [2,3], siendo más complejos al involucrar el conocimiento y las capacidades de raciocinio en los objetos, permitiendo tomar decisiones o comportamientos sociales por medio de la conciencia del contexto [7]. Cabe anotar que hace falta el modelamiento de otros contextos como el dominio de interacción (medio ambiente, agricultura, salud, etcétera), requisitos de dominio de usuarios (personas empresa, reglamentación y normatividad) y mecanismos de interacción inteligente (interacción dinámica y creación de nuevos servicios). Este articulo comprende los conceptos básicos a tener en cuenta en la interacción semántica de objetos (cosas) de la WoT, evidenciando el estado actual del conocimiento, presentando un análisis detallado de las áreas de desarrollo identificadas y las conclusiones.

\section{Metodología}

Se utilizó la investigación documental propuesta por [8], que consta de cuatro fases: Preparatoria, Descriptiva, Interpretación por núcleos temáticos y Construcción teórica global, teniendo como núcleos temáticos: Web Semántica de las Cosas, Internet de las Cosas e Interacción Semántica y realizando un estudio comparativo con 38 fichas bibliográficas de 60 referencias totales analizadas.

\section{Estado del arte}

A partir de la metodología definida en esta investigación se presenta a continuación el estado actual del conocimiento relacionado con la interacción semántica de las cosas:

\section{Conceptos Base de Interacción Semántica de las Cosas}

Las aplicaciones IOT y las ontologías permiten estandarizar técnicas de manipulación, reutilización e integración de las medidas obtenidas por los sensores dando paso a la Web of Things (WoT) que crea conocimiento a partir de la identificación y gestión de las anotaciones semánticas (estructuras de información, generalmente con formatos de intercambio de conocimiento bien conocidos en la web como XML, RDF y OWL), de los objetos de la loT, las entidades con las cuales están relacionados y el contexto de interacción, permitiendo obtener información semántica de los objetos inteligentes y de sus relaciones con el entorno al mejorar el funcionamiento de las máquinas [9]. Cabe anotar que para gestionar el conocimiento es necesario trabajar con objetos inteligentes, los cuales, tienen capacidades de almacenamiento, procesamiento y comunicación embebidas en los dispositivos, creando puertas de enlace inteligentes [10], como puntos intermedios en la red semántica; estos son capaces de recopilar información de dichos objetos. 
Se han creado plataformas middlewares que permiten resolver problemas de heterogeneidad profunda apuntando a modelos que utilizan la arquitectura REST (Representational State Transfer) y/o SOA (Service-Oriented Architecture)[11], teniendo en cuenta que el acceso transparente a los servicios de las "cosas" es más intuitivo en arquitecturas REST y para servicios de negocios es más intuitivo SOA, el cual es importante para masificar el uso de servicios de la WoT. Además, estas plataformas implementan protocolos de comunicaciones como CoAP (Protocolo de aplicación restringida), MQTT (Transporte de telemetría de mensajes en cola) y XMPP (Protocolo de presencia y mensajería extensible) para su montaje en dispositivos livianos. Exponen los datos y la información de metadatos utilizando formatos estándar como: JSON, XML, CSV y XDR. Hay cuatro tipos de estas aplicaciones: Middlewares, Servidores Web IOT, Productos empresariales y Computación en la nube, los cuales juegan un papel importante en el desarrollo de la loT, ya que su diseño está más orientado a una visión de los sensores como proveedores de servicios gestionados a través de Big Data, área que propone nuevos retos para manejar la gran cantidad de datos que se generan a partir de los sensores. Entre las principales plataformas existentes tenemos a SenaaS y Sensor-Cloud, la cual usa SensorML para describir los metadatos de los sensores físicos, empaquetando los mismos en una representación digital que los usuarios pueden combinar.

También se han creado repositorios de conocimiento basados en ontologías [12,13] para el acceso a datos a través de los objetos. Se han implementado diferentes estrategias para almacenar y procesar el conocimiento proveniente de los sensores de la loT y las soluciones se orientan al desarrollo de ontologías para el manejo de: capacidades, observaciones de los eventos, categorización, comportamiento social, servicios, conocimiento, estimación, conversión y contexto. Se destaca el trabajo presentado por el grupo denominado W3C Semantic Sensor Network Incubator group [14] los cuales han creado una ontología llamada Semantic Sensor Network (SSN) y la última actualización Sensor-Observation-Sampling-Actuator Ontology (SOSA), que permite describir las capacidades de los sensores, el proceso de medición y las observaciones resultantes en una red de sensores semánticos. Esta ontología ha reutilizado los demás estándares existentes en la representación de sensores, tales como SensorML y O\&M del Open Geospatial Consortium (OGC) y de estándares definidos por el proyecto Sensor Web Enablement (SWE).

Finalmente, las tecnologías utilizadas por los trabajos recientes para la implementación de la interacción semántica entre objetos se pueden resumir en: middlewares orientados a servicios de sensores [15], middlewares orientados a microservicios [16], arquitecturas orientadas a eventos [17], desarrollo de agentes inteligentes para objetos inteligentes [18] y basados en ontologías y mensajes en redes sociales [19].

- Análisis de la Interoperabilidad Semántica por Áreas de Investigación Identificadas: A partir del análisis bibliográfico, se pudo identificar una evolución gradual del desarrollo de la interoperabilidad semántica de las cosas, clasificando los trabajos en tres grandes áreas de desarrollo:

- Arquitecturas loT: Las investigaciones aportan en el diseño de alto nivel del ecosistema, qué debería existir para tener un entorno de interacción semántica loT coincidiendo en capas bien definidas con funcionalidades encapsuladas. Entre los principales conceptos aportados están: Gateways IoT, Middlewares, los protocolos de comunicación, servicios e interfaces de realidad aumentada para la IOT.

- Web Semántica de las Cosas: Intenta dar a los objetos de la loT capacidades semánticas, utilizando técnicas de anotación semántica, ontologías y sistemas orientados a servicios. Entre los principales conceptos aportados están: Anotaciones semánticas, modelamiento del contexto, Middlewares Semánticos y Ontologías sensor como la SSN [20]. 
- Comportamiento Social e Inteligente de las cosas: Implementan mecanismos de inteligencia computacional con el fin de representar el conocimiento e incorporar la capacidad de tomar decisiones a los objetos inteligentes de la IOT. Entre los conceptos clave aportados están: Red social de Objetos Inteligentes, Interacción Social de Objetos, Comportamiento Inteligente de objetos IoT y Representación del conocimiento.

A continuación, se realiza un estudio comparativo de investigaciones por las áreas identificadas, con el fin de encontrar similitudes, tendencias y técnicas utilizadas en el desarrollo de cada área.

\section{Arquitecturas loT}

En general, se plantean arquitecturas basadas en capas como se presenta en la Tabla 1, mostrando un estudio comparativo con el fin de obtener los consensos entre capas y los principales modelos identificados para crear arquitecturas en la loT.

Tabla 1 Investigaciones sobre las propuestas de arquitectura loT.

\begin{tabular}{|c|c|c|c|c|c|c|c|c|c|c|c|}
\hline \multirow{3}{*}{ Proyecto } & \multicolumn{7}{|c|}{ Capas identificadas } & \multirow{2}{*}{\multicolumn{4}{|c|}{ Modelos }} \\
\hline & \multicolumn{3}{|c|}{ Capa de percepción } & \multicolumn{2}{|c|}{ Capa de Red } & \multicolumn{2}{|c|}{$\begin{array}{c}\text { Capa de } \\
\text { aplicación }\end{array}$} & & & & \\
\hline & $\begin{array}{l}\frac{8}{\frac{3}{n}} \\
\frac{2}{2} \\
\frac{0}{0} \\
\frac{0}{0}\end{array}$ & 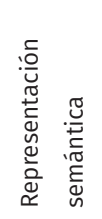 & 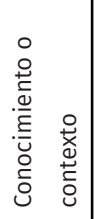 & 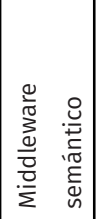 & 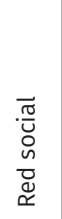 & 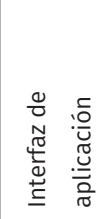 & 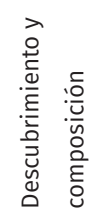 & 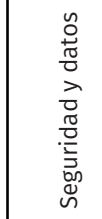 & 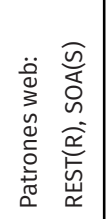 & 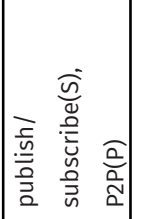 & 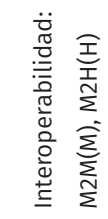 \\
\hline $\begin{array}{l}\text { Dominique Guinard, } \\
\text { Floerkemeier, \& Sarma } \\
\text { [21] }\end{array}$ & $x$ & $x$ & & & $x$ & $x$ & & $\mathrm{x}$ & $\mathrm{R}$ & & $\mathrm{H}$ \\
\hline $\begin{array}{l}\text { Kosmatos and Tselikas } \\
\text { [11] }\end{array}$ & $x$ & $x$ & & $\mathrm{x}$ & $x$ & & $x$ & & $\mathrm{x}$ & S & M \\
\hline Vega-Barbas, et al. [22] & $x$ & $x$ & $x$ & & $x$ & $x$ & $x$ & $x$ & S & & M \\
\hline Heesuk, et al. [23] & $x$ & $x$ & $x$ & & $x$ & $x$ & $x$ & & & & $\mathrm{H}$ \\
\hline Wan, et al. [17] & $x$ & $x$ & $x$ & $x$ & & $x$ & $x$ & & $x$ & & M \\
\hline Perera, et al. [24] & $x$ & $x$ & $x$ & $x$ & & & & & S & & $\mathrm{H}$ \\
\hline Desai, et al. [10] & $x$ & $x$ & $x$ & $x$ & & & $x$ & $x$ & s & $x$ & $\mathrm{H}$ \\
\hline $\begin{array}{l}\text { Mahmuod, Yousuf, Aloul } \\
\text { \& Zualkernan [25] }\end{array}$ & $x$ & & $x$ & & & $x$ & & $x$ & & & $\mathrm{H}$ \\
\hline Chiang \& Zhang [26] & $x$ & & & & & $x$ & & $x$ & & & $\mathrm{H}$ \\
\hline $\begin{array}{l}\text { Yang, Wu, Yin, Li, \& } \\
\text { Zhao [27] }\end{array}$ & $\mathrm{X}$ & & $x$ & $x$ & & $\mathrm{X}$ & & $x$ & & & \\
\hline \begin{tabular}{|l|} 
Yassein, Shatnawi, \\
Aljwarneh, \& Al-Hatmi \\
[28]
\end{tabular} & $\mathrm{X}$ & & & & & $x$ & & $x$ & $\mathrm{R}$ & & M \\
\hline $\begin{array}{l}\text { Lo, Liew, Tey, \& } \\
\text { Mekhilef [29] }\end{array}$ & $\mathrm{X}$ & & $\mathrm{X}$ & $X$ & & $\mathrm{X}$ & & $X$ & & & $M$ \\
\hline
\end{tabular}

Fuente: Elaboración propia 
En general se pueden identificar tres capas básicas o principales 1 capa de percepción que comprende los objetos físicos, la representación semántica, el contexto o conocimiento, seguida a esta capa se encuentra la capa de red que incluye temas como middleware y red social y por último la capa de aplicación que comprende temas de aplicación, descubrimiento y composición:

- Capa de percepción: Comprende los recursos físicos con los que tiene contacto la aplicación: sensores, actuadores, objetos inteligentes entre otros, que generan información del entorno de estudio a través de diversos protocolos hardware, software y comunicación entre sensores como: MQTT, XMPP (modelo publicador / suscriptor) o COAP (modelo REST). Con respecto a los protocolos de red, se trabaja sobre TCP/IP; sin embargo, se está implementando el protocolo 6LOWpan, por ser más ligero en los dispositivos de la loT resolviendo disponibilidad de direcciones.

- Capa de red: Permite realizar un puente entre la capa de percepción y la capa de aplicación, desarrollando plataformas software que permiten abstraer las complejidades de conexión de los dispositivos de la capa de percepción, con una interfaz única de acceso a las aplicaciones, que describe los sensores mediante la capa de representación basado en estilos arquitectónicos como REST o SOA y módulos sociales.

- Capa de aplicación: Los principales módulos identificados son: Contexto[30], Descubrimiento y composición de servicios. Esta capa realiza la entrega y ejecuta las acciones pertinentes a la aplicación situando las interfaces de los usuarios, que pueden estar destinadas a desplegarse en diferentes dispositivos como computadores personales, teléfonos inteligentes, tabletas y otras aplicaciones que utilizan sus datos como entradas. Con respecto a las interfaces interactivas se tienen tecnologías web como: HTML5, AJAX y Mashups físicos, pero ya se está incursionando en la integración de la realidad aumentada como elemento importante de interacción M2H[31].

Además, se encontró un módulo transversal a las demás capas y muy importante, el de seguridad [32], que contiene protocolos de autenticación y autorización para el acceso a los datos, metadatos y servicios de los sensores que también están utilizando cifrado de datos y otros protocolos de seguridad como OAuth2.

Las brechas existentes que se pueden identificar en esta área son:

- Se encontró un vacío respecto a la información contextual proveniente de los datos capturados de los sensores ya que los mismos carecen de semántica para desarrollar interfaces adecuadas a las personas. Normalmente, se implementa con modelos conceptuales basados en ontologías, pero aún no se ha unificado esta información para hacerla completamente interoperable.

- El trabajo desarrollado en privacidad y seguridad está relacionado con técnicas de compresión y cifrado de datos, manejo de contraseñas y seguridad otorgada por las redes sociales; sin embargo, requieren un trabajo más holístico y elaborado para no ser un obstáculo para el uso de los objetos con capacidades semánticas en las actividades diarias de las mismas.

- Las arquitecturas que se enfocan en describir con mayor detalle la interacción M2M aún son escazas y la mayoría de los estudios plantean mecanismos de envío de mensajes entre dispositivos, pero poco en la cooperación de acuerdo con el contexto identificado, lo cual requiere un mayor grado de complejidad.

1 Se omiten las referencias de los siguientes párrafos dado que cada referencia en la Tabla 1 aportan a las diferentes dimensiones analizadas. 


\section{Web Semántica de las Cosas}

Para construir conocimiento alrededor de objetos, se ha propuesto la creación de repositorios de conocimiento basados en ontologías. Tabla 2 mediante la implementación de diferentes estrategias para almacenar y procesar el conocimiento de los sensores de loT.

Tabla 2 Investigación relacionada con la interoperabilidad semántica.

\begin{tabular}{|c|c|c|c|c|c|c|c|c|c|c|}
\hline \multirow[t]{2}{*}{ Proyecto } & \multicolumn{10}{|c|}{ Conceptos y técnicas semánticas } \\
\hline & 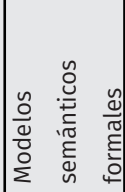 & 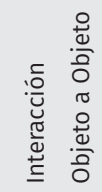 & 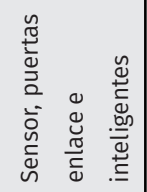 & 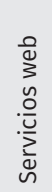 & 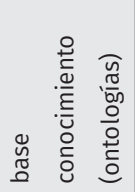 & 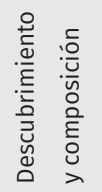 & 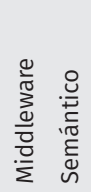 & 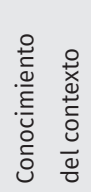 & 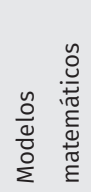 & $\begin{array}{ll}\frac{0}{0} & \\
\frac{\pi}{0} & \\
\frac{0}{U} & \tilde{0} \\
0 & 0 \\
0 & 0 \\
0 & 0 \\
0 & n \\
3 & 0\end{array}$ \\
\hline $\begin{array}{l}\text { Kostelník, et al. } \\
\text { [33] }\end{array}$ & $x$ & & $x$ & $x$ & $x$ & $x$ & $x$ & $x$ & $x$ & \\
\hline $\begin{array}{l}\text { Hachem, et al. [12], } \\
\text { Teixeira, et al. [34] }\end{array}$ & $x$ & & & $x$ & $\mathrm{x}$ & $x$ & $x$ & & $x$ & \\
\hline $\begin{array}{l}\text { Vega-Barbas, et } \\
\text { al. [35] }\end{array}$ & $x$ & $x$ & $x$ & $x$ & $X$ & $x$ & $x$ & $x$ & & \\
\hline Biamino [13] & $x$ & & $x$ & & $x$ & & & $x$ & $x$ & $x$ \\
\hline Wang, et al. [36] & $x$ & & & & $x$ & & & & & \\
\hline Yao [37] & $x$ & $x$ & & & $x$ & & & & $x$ & \\
\hline Pintus, et al. [6] & $x$ & & & & & $x$ & $x$ & & & $x$ \\
\hline Razzak [38] & $x$ & $x$ & $x$ & & $x$ & & & $x$ & & \\
\hline $\begin{array}{l}\text { Aggarwal and } \\
\text { Abdelzaher [39] }\end{array}$ & & & $x$ & & & & & $x$ & & $x$ \\
\hline Desai, et al. [10] & $x$ & $x$ & $x$ & $x$ & $x$ & & $x$ & & & \\
\hline Perera, et al. [24] & $x$ & & & & $x$ & $x$ & & $x$ & & \\
\hline $\begin{array}{l}\text { Androcec \& Vrcek } \\
{[40]}\end{array}$ & $x$ & $x$ & $x$ & & & & $x$ & & & $x$ \\
\hline \begin{tabular}{|l} 
De, Zhou, \\
Moessner [41]
\end{tabular} & $x$ & & & & $x$ & & & $x$ & & \\
\hline $\begin{array}{l}\text { Akanbi \& Masinde } \\
{[42]}\end{array}$ & & $x$ & $x$ & & $x$ & & $x$ & & & \\
\hline \begin{tabular}{|l|} 
Thota \& \\
Mahalakshmi [43]
\end{tabular} & & & $x$ & & & $x$ & & & & \\
\hline $\begin{array}{l}\text { Gil, Johnsson, } \\
\text { Mora, \& Szymański } \\
{[44]}\end{array}$ & $x$ & & & $x$ & $x$ & $x$ & & & & \\
\hline
\end{tabular}

Fuente: Elaboración propia 
En la Tabla 2 se hizo un estudio comparativo de las principales características que implementaron los proyectos enfocados en la interoperabilidad semántica para la loT. En general, se busca un modelo semántico que capture el contexto-conocimiento por medio de ontologías de dominio [45] y a la vez, permita definir los mecanismos de interacción ente los objetos de la IoT. Los modelos son implementados en Gateways semánticos [46], como agregadores naturales de los dispositivos en una red o entorno de interacción. De igual forma la combinación entre la Web semántica y fuentes de datos es fundamental para la evolución de la interoperabilidad debido a que permite extraer, integrar y generar conocimiento [47]. Con respecto a los servicios, se busca que sean provistos a través de servicios web, con procesos de descubrimiento y composición de estos. Las redes sociales de objetos se presentan como la punta del iceberg de este campo de desarrollo. Finalmente, se reconoce que todos los mecanismos de interacción e interoperabilidad deben estar estandarizados [41], para permitir la evolución de este tipo de sistemas.

Las brechas existentes en esta área que se pueden identificar son:

- La creación de modelos formales semánticos, con el fin de encontrar un mecanismo adecuado para aprovechar la información y el conocimiento inferido de los objetos.

- El modelamiento del contexto y de la conciencia en donde se identifican elementos de contexto, pero hasta ahora se está estudiando cómo implementarlo adecuadamente.

- La indexación semántica lo cual podría ser un buen punto de partida para implementar esta funcionalidad.

- La web semántica normalmente utiliza ontologías para representar el conocimiento y realizar procesos básicos de razonamientos sobre ese conocimiento, utilizando reglas básicas de lógica de primer orden. Es necesario desarrollar investigaciones que involucren algoritmos basados en otras técnicas de inteligencia artificial para crear interacciones entre los diferentes sensores conectados a la red, con el objetivo de optimizar procesos y producir información, conocimiento y sabiduría en aplicaciones del mundo real.

\section{Comportamiento social e Inteligente de las Cosas}

Para desarrollar comportamientos sociales, se debe enfocar en capacidades inteligentes de los objetos, elementos que requieren el aporte de disciplinas como aprendizaje ontológico, inteligencia artificial y aprendizaje de máquina. El desarrollo completo de esta área dependerá del mejoramiento en la capacidad computacional que puede darse de diversas maneras dentro de ellas por medio de mejoras en el desarrollo del comportamiento inteligente de los objetos y el avance de la nanotecnología y permitiendo el abaratamiento de sus costos, lo cual es una tendencia actual. La Tabla 3 hace una comparación de los principales estudios encontrados que se relacionan con esta área. 
Tabla 3 Investigación relacionada con el comportamiento social e inteligente.

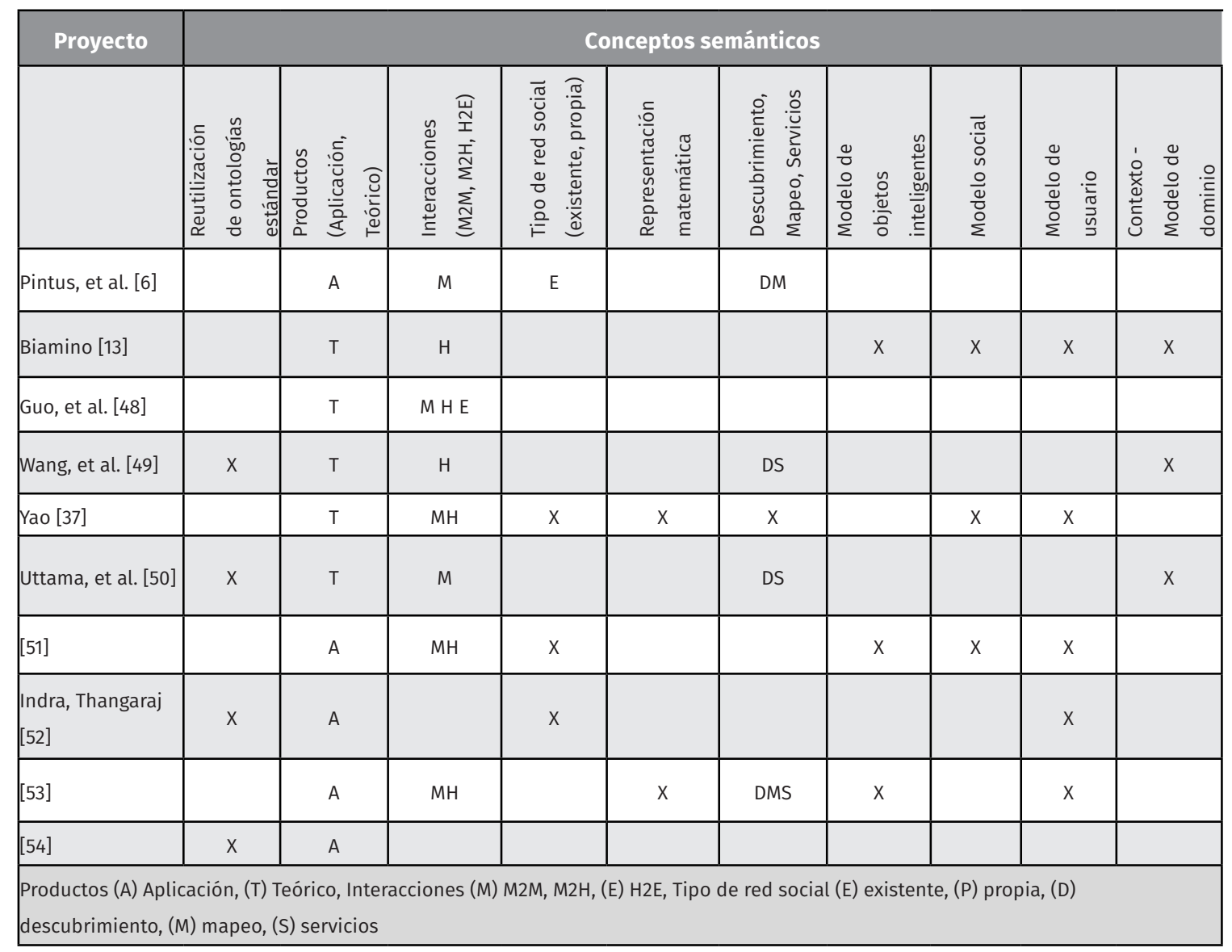

Fuente: Elaboración propia

Los principales conceptos identificados en el análisis comparativo de los estudios en el campo del comportamiento inteligente de los objetos son la reutilización de estándares ampliamente aceptados por la comunidad investigativa como: SSN, Geonames, OWL-S o Quantity Kinds and Units. Por otro lado, las investigaciones aportan con modelamientos de redes sociales de objetos, los cuales definen interacciones a través de modelos de descubrimiento de servicios y composición de estos. Las propuestas más relevantes, definen objetos sociales sensibles al contexto [6,55], modelos de recomendación de amigos [37] y tecnologías para incrustar inteligencia en los objetos de la IOT o W2T Guo, et al. [48]. El surgimiento de las investigaciones involucra algoritmos de inteligencia artificial como arboles de decisión y redes neuronales convolucionales permitiendo encontrar relaciones entre los datos obtenidos de los diferentes objetos que suministran información del entorno, evaluando ciertos criterios y mejorando los procesos. Estas investigaciones han sido aplicadas en diferentes campos del conocimiento logrando resultados positivos y obteniendo productos inteligentes como: Calcetines inteligentes [56], asistentes virtuales con conciencia emocional [57], diseños ambientales adaptativos [58], estetoscopio inteligente [59], guantes inteligentes [60] y demás productos que incluyen otras tecnologías para su aplicación. 
Las brechas existentes en esta área que se pueden identificar son:

- Los conceptos de redes sociales de objetos aún son más conceptuales y aún no es claro si se deben conectar a las redes sociales existentes o crear una red propia de objetos.

- Muy pocos estudios abordan las características que debe tener la interacción entre objetos (modelo social) en una red social de objetos que se colaboran.

- En cuando a las redes sociales y los objetos, aún no se logran compartir los objetos e interactuar con ellos efectivamente, siendo necesarias la programación de relaciones y movimiento de código fuente a dispositivos por parte del usuario.

- El razonamiento y la inteligencia de los objetos está por desarrollar, ya que hasta ahora se está buscando como almacenar el conocimiento adecuadamente que permita su uso y racionamiento. Las ontologías pueden ser una solución, pero deben realizarse adecuadamente para evitar proliferación de ontologías incorrectas, generando errores sin poder ser reutilizadas.

- La Wisdom Web of Things - W2T, necesita interdisciplinariedad para encontrar un modelo escalable que avance en las capacidades de procesamiento con el fin de embeber más objetos con inteligencia.

- La inteligencia artificial de las cosas (Artificial Intelligence of Things - AloT), debe aplicar algoritmos de aprendizaje de máquina o algoritmos de inteligencia artificial en los mismos dispositivos IoT (fog computing), para producir información, conocimiento y sabiduría de los datos enviados por diferentes sensores, servicios de cómputo en la nube que permitan aplicar modelos entrenados de algoritmos de aprendizaje que generen los reportes o evaluaciones de diferentes situaciones en un ambiente real: housing, healthcare, food, Smart cities, entren otros.

\section{Conclusiones}

Se identificó una nueva área de investigación conocida como Inteligencia Artificial de la Cosas (AloT, por sus siglas en inglés), la cual permitirá el análisis de gran cantidad de información que es capturada de los sensores conectados a internet, obteniendo información valiosa a través de algoritmos de inteligencia artificial avanzando con ayuda de Big Data y el crecimiento de las capacidades hardware de los servidores para cómputo en la nube.

Este artículo analizó y evaluó proyectos de investigación en el área de la interacción semántica de la WoT y la creación de la SWoT, con el fin de encontrar las tecnologías, y modelos al momento de resolver los retos de la incorporación de inteligencia a los sensores de la WoT, así como también identificar los retos, falencias y aciertos de las soluciones analizadas.

\section{Referencias bibliográficas}

1. N. Sekkal, S. M. Benslimane, M. Mrissa, C. Y. Park, and B. Boudaa, "Proactive and reactive context reasoning architecture for smart web services," (in English), International Journal of Data Mining, Modelling and Management, Article vol. 12, no. 1, pp. 1-27, 2020.

2. M. Imran, S. Jabbar, N. Chilamkurti, and J. J. P. C. Rodrigues, "Enabling technologies for Social Internet of Things," Future Generation Computer Systems, vol. 92, pp. 715-717, 2019. 
3. S. Rho and Y. Chen, "Social Internet of Things: Applications, architectures and protocols," Future Generation Computer Systems, vol. 92, pp. 959-960, 2019.

4. M. Blackstock, R. Lea, and A. Friday, "Uniting online social networks with places and things," presented at the Proceedings of the Second International Workshop on Web of Things, San Francisco, California, 2011.

5. L. Atzori, A. Iera, G. Morabito, and M. Nitti, "The Social Internet of Things (SIoT) - When social networks meet the Internet of Things: Concept, architecture and network characterization," Computer Networks, vol. 56, no. 16, pp. 3594-3608, 2012.

6. A. Pintus, D. Carboni, and A. Piras, "Paraimpu: a platform for a social web of things," presented at the Proceedings of the 21st international conference companion on World Wide Web, Lyon, France, 2012.

7. C. Perera, P. Jayaraman, A. Zaslavsky, P. Christen, and D. Georgakopoulos, "Context-Aware Dynamic Discovery and Configuration of 'Things' in Smart Environments," Big Data and Internet of Things: A Roadmap for Smart Environments, Studies in Computational Intelligence book series, Springer Berlin Heidelberg, 2014.

8. C. E. Serrano, "Modelo para la Investigación Documental," in Modelo Integral para el Profesional en Ingeniería, U. d. Cauca, Ed. Popayán, 2008.

9. K. N. Prashanth Kumar, V. Ravi Kumar, and K. Raghuveer, "A Survey on Semantic Web Technologies for the Internet of Things," in International Conference on Current Trends in Computer, Electrical, Electronics and Communication, CTCEEC 2017, ed: Institute of Electrical and Electronics Engineers Inc., 2018, pp. 316-322.

10. P. Desai, A. Sheth, and P. Anantharam, "Semantic Gateway as a Service architecture for IoT Interoperability," arXiv.org, p. 16, 10/2014. Accessed on: 16/11/2014

11. E. Kosmatos and N. Tselikas, Integrating RFIDs and Smart Objects into a Unified Internet of Things Architecture. scirp.org, 2011.

12. S. Hachem, T. Teixeira, and V. Issarny, "Ontologies for the Internet of Things," Proceedings of the 8th Middleware Doctoral Symposium, 2011.

13. G. Biamino, "A Semantic Model for Socially Aware Objects," Advances in Internet of Things, 2012.

14. M. Compton et al., "The SSN ontology of the W3C semantic sensor network incubator group," Web Semantics: Science, 2012.

15. H. M. Chen, H. L. Shi, M. Li, and L. Cui, "Service Middleware for Internet of Things: Challenges and Approaches," (in Chinese), Jisuanji Xuebao/Chinese Journal of Computers, Review vol. 40, no. 8, pp. 1725-1749, 2017.

16. A. Benayache, A. Bilami, S. Barkat, P. Lorenz, and H. Taleb, "MsM: A microservice middleware for smart WSN-based IoT application," (in English), Journal of Network and Computer Applications, Article vol. 144, pp. 138-154, 2019.

17. J. Wan, D. Li, C. Zou, and K. Zhou, “M2M Communications for Smart City: An Event-Based Architecture," in Computer and Information Technology (CIT), 2012 IEEE 12th International Conference on, 2012, pp. 895-900.

18. D. Ajitomi, H. Kawazoe, K. Minami, and N. Esaka, "A Cost-Effective Method to Keep Availability of Many Cloud-Connected Devices," 2015, pp. 1-8: Institute of Electrical and Electronics Engineers Inc.

19. C. Zhang, C. Cheng, and Y. Ji, “Architecture design for social web of things," presented at the Proceedings of the 1st International Workshop on Context Discovery and Data Mining, Beijing, China, 2012. 
20. M. Compton et al., "The SSN ontology of the W3C semantic sensor network incubator group," Web Semantics: Science, Services and Agents on the World Wide Web, no. 0, 2012.

21. D. Guinard, C. Floerkemeier, and S. Sarma, "Cloud computing, rest and mashups to simplify rfid application development and deployment," Proceedings of the 2nd International Workshop on the Web of Things (WoT 2011), 2011.

22. M. Vega-Barbas, D. Casado-Mansilla, M. A. Valero, D. Lopez-de-Ipina, J. Bravo, and F. Florez, "Smart Spaces and Smart Objects Interoperability Architecture (S3OiA)," in Innovative Mobile and Internet Services in Ubiquitous Computing (IMIS), 2012 Sixth International Conference on, 2012, pp. 725-730.

23. S. Heesuk, H. Seungwook, and L. Dongman, "Contextual Information Provision on Augmented Reality with IOT-Based Semantic Communication," in Ubiquitous Virtual Reality (ISUVR), 2012 International Symposium on, 2012, pp. 46-49.

24. C. Perera, A. Zaslavsky, C. H. Liu, M. Compton, P. Christen, and D. Georgakopoulos, Sensor search techniques for sensing as a service architecture for the internet of things. ieeexplore.ieee.org, 2013.

25. R. Mahmoud, T. Yousuf, F. Aloul, and I. Zualkernan, "Internet of things (IOT) security: Current status, challenges and prospective measures," 2016, pp. 336-341: Institute of Electrical and Electronics Engineers Inc.

26. M. Chiang and T. Zhang, "Fog and IOT: An Overview of Research Opportunities," (in English), IEEE Internet of Things Journal, Review vol. 3, no. 6, pp. 854-864, 2016, Art. no. 7498684.

27. Y. Yang, L. Wu, G. Yin, L. Li, and H. Zhao, "A Survey on Security and Privacy Issues in Internet-of-Things," (in English), IEEE Internet of Things Journal, Article vol. 4, no. 5, pp. 1250-1258, 2017, Art. no. 7902207.

28. M. B. Yassein, M. Q. Shatnawi, S. Aljwarneh, and R. Al-Hatmi, "Internet of Things: Survey and open issues of MQTT protocol," 2018, vol. 2018-January, pp. 1-6: Institute of Electrical and Electronics Engineers Inc.

29. S. K. Lo, C. S. Liew, K. S. Tey, and S. Mekhilef, "An interoperable component-based architecture for data-driven IoT system," (in English), Sensors (Switzerland), Article vol. 19, no. 20, 2019, Art. no. 4354.

30. C. Perera, P. Jayaraman, A. Zaslavsky, P. Christen, and D. Georgakopoulos, "Context-Aware Dynamic Discovery and Configuration of 'Things' in Smart Environments," Big Data and Internet of Things: A Roadmap for Smart Environments, Studies in Computational Intelligence book series, Springer Berlin Heidelberg, 2013.

31. A. Mishra, S. Karmakar, A. Bose, and A. Dutta, "Design and development of loT-based latencyoptimized augmented reality framework in home automation and telemetry for smart lifestyle," Journal of Reliable Intelligent Environments, Article 2020.

32. J. Lin, W. Yu, N. Zhang, X. Yang, H. Zhang, and W. Zhao, "A Survey on Internet of Things: Architecture, Enabling Technologies, Security and Privacy, and Applications," IEEE Internet of Things Journal, Article vol. 4, no. 5, pp. 1125-1142, 2017, Art. no. 7879243.

33. P. Kostelník, M. Sarnovský, and K. Furdík, "The Semantic Middleware for Networked Embedded Systems Applied in the Internet of Things and Services Domain," Scalable Computing: Practice and Experience, vol. 12, no. 3, 2011.

34. T. Teixeira, S. Hachem, V. Issarny, and N. Georgantas, "Service oriented middleware for the internet of things: A perspective," in Towards a Service-Based Internet 4th European Conference, ServiceWave 2011, Poznan, Poland., 2011, 1 cites: http://scholar.google.com/scholar?cites=3865235688391678588\&as_ $\mathrm{sdt}=2005 \&$ sciodt $=0,5 \& \mathrm{hl}=$ en\&num $=100$ 
35. M. Vega-Barbas, D. Casado-Mansilla, and J. R. Velasco, "S3OiA: Propuesta de Arquitectura para la Interoperabilidad en la Internet de las Cosas," in X Jornadas de Ingeniería Telemática - JITEL 2011, Santander, España, 2011, Query date: 2012-09-18.

36. W. Wang, S. De, R. Toenjes, E. Reetz, and K. Moessner, “A Comprehensive Ontology for Knowledge Representation in the Internet of Things," in IEEE 11th International Conference onTrust, Security and Privacy in Computing and Communications (TrustCom-2012), 2012, pp. 1793-1798.

37. L. Yao, "A Propagation Model for Integrating Web of Things and Social Networks," presented at the Service-Oriented Computing - ICSOC 2011 Workshops, 2012/01/01, 2012. Available: DOI: http://dx.doi. org/10.1007/978-3-642-31875-7_28

38. F. Razzak, "The role of semantic web technologies in smart environments," porto.polito.it, 2013.

39. C. Aggarwal and T. Abdelzaher, "Social Sensing," in Managing and Mining Sensor Data, 2013.

40. D. Androcec and N. Vrcek, “Thing as a service interoperability: Review and framework proposal," 2016, pp. 309-316: Institute of Electrical and Electronics Engineers Inc.

41. S. De, Y. Zhou, and K. Moessner, "Ontologies and context modeling for the Web of Things," in Managing the Web of Things: Linking the Real World to the Web: Elsevier Inc., 2017, pp. 3-36.

42. A. K. Akanbi and M. Masinde, "Semantic interoperability middleware architecture for heterogeneous environmental data sources," 2018: Institute of Electrical and Electronics Engineers Inc.

43. S. K. Thota and T. S. Mahalakshmi, "A semantic based terms relation research for classification in web information mining," (in English), International Journal of Recent Technology and Engineering, Article vol. 8, no. 2, pp. 5275-5280, 2019.

44. [44] D. Gil, M. Johnsson, H. Mora, and J. Szymański, "Review of the Complexity of Managing Big Data of the Internet of Things," (in English), Complexity, Review vol. 2019, 2019, Art. no. 4592902.

45. M. Kolchin et al., "Ontologies for web of things: A pragmatic review," vol. 518, P. Klinov and D. Mouromtsev, Eds., ed: Springer Verlag, 2015, pp. 102-116.

46. D. C. Yaccirema Vargas, “Arquitectura de Interoperbilidad De Dispositivos Físicos Para el Internet de la Cosas (IOT)," PhD Doctoral Thesis, Departamento de Comunicaciones, Universidad Politecnica de Valencia, Valencia, España, 2019.

47. S. Mishra and S. Jain, "Ontologies as a semantic model in IoT," International Journal of Computers and Applications, Article vol. 42, no. 3, pp. 233-243, 2020.

48. B. Guo, D. Zhang, Z. Yu, Y. Liang, Z. Wang, and X. Zhou, “From the internet of things to embedded intelligence," (in English), World Wide Web, pp. 1-22, 2012.

49. W. Wang, D. Suprama, G. Cassar, and K. Moessner, "Knowledge Representation in the Internet of Things: Semantic Modelling and its Applications," Automatika - Journal for Control, Measurement, Electronics, Computing and Communications, vol. 54, pp. 388-400, 2013.

50. A. Uttama, C. Sarkar, V. Prasad, and A. Rahim, “A unified semantic knowledge base for IoT," in Internet of Things (WF-IOT), 2014 IEEE World Forum on, ed, 2014, pp. 575-580.

51. N. Junior, R. P. C. Nascimento, E. Souto, R. Aschoff, and W. Sousa, "A visual approach for the definition of behavior in environments for internet of things," 2017, pp. 381-388: Association for Computing Machinery, Inc.

52. R. Indra and M. Thangaraj, "An integrated recommender system using semantic web with social tagging system," (in English), International Journal on Semantic Web and Information Systems, Article vol. 15, no. 2, pp. 47-67, 2019. 
53. S. Ojagh, M. R. Malek, S. Saeedi, and S. Liang, "A location-based orientation-aware recommender system using IOT smart devices and Social Networks," (in English), Future Generation Computer Systems, Article vol. 108, pp. 97-118, 2020.

54. Z. Sayah, O. Kazar, B. Lejdel, A. Laouid, and A. Ghenabzia, "An intelligent system for energy management in smart cities based on big data and ontology," (in English), Smart and Sustainable Built Environment, Article 2020.

55. A. Pintus, D. Carboni, and A. Piras, "The anatomy of a large scale social web for internet enabled objects," presented at the Proceedings of the Second International Workshop on Web of Things, San Francisco, California, 2011.

56. Z. Zhang, T. He, M. Zhu, Q. Shi, and C. Lee, "Smart Triboelectric Socks for Enabling Artificial Intelligence of Things (AloT) Based Smart Home and Healthcare," 2020, vol. 2020-January, pp. 80-83: Institute of Electrical and Electronics Engineers Inc.

57. P. S. Chiu, J. W. Chang, M. C. Lee, C. H. Chen, and D. S. Lee, “Enabling intelligent environment by the design of emotionally aware virtual assistant: A case of smart campus," (in English), IEEE Access, Article vol. 8, pp. 62032-62041, 2020, Art. no. 9050793.

58. C. C. Chen, "Incorporating smart technologies and resilience into healthy living environment designs," (in Chinese), Journal of Nursing, Article vol. 66, no. 3, pp. 23-28, 2019.

59. Y. J. Lin et al., "An intelligent stethoscope with ECG and heart sound synchronous display," 2019 , vol. 2019-May: Institute of Electrical and Electronics Engineers Inc.

60. M. Zhu et al., "Haptic-feedback smart glove as a creative human-machine interface (HMI) for virtual/ augmented reality applications," Science Advances, Article vol. 6, no. 19, 2020, Art. no. eaaz8693. 